\title{
INDICATION OF AMPUTATION AFTER TUMORAL ARTHROPLASTY
}

doi: 10.2478/rojost-2018-0020

\author{
A. Lupu 12, R. Ene 12, A. Cursaru12, D. Anghelescu1, E. Popescu1, C. Cîrstoiv ${ }^{12}$ \\ ${ }^{1}$ University Emergency Hospital, Bucharest, Romania \\ 2"Carol Davila" University of Medicine and Pharmacy, Bucharest, Romania
}

Introduction. After a time when amputation was the only treatment option for musculoskeletal malignancies, a major breakthrough was the tumoral arthroplasty with limb preservation.

Material and method. The study included a group of 28 patients, of whom 20 had pelvic limb tumor formation. Malignant bone tumors were present in 18 patients and benign tumors in 10 patients. The most commonly encountered was osteosarcoma in 12 patients, Ewing sarcoma in 4 patients and giant cell tumor in 3 patients. Patient follow-up was conducted within 4 years (with an average of 3-5 years).

Results. 5 of our patients developed pulmonary metastases 8 months after surgery and, for 4 of the patients, pulmonary determinations were extirpated without subsequent relapse. The survival rate was $75 \%$ at the last follow-up ( 6 of 12 patients with osteosarcoma, 1 of 4 patients with Ewing's sarcoma has died), and $32 \%$ had local tumor recurrence. The infection remains an inherent danger by using implants in immunosuppressed patients. $18 \%$ of the patients had amputations secondary to long-term complications involving the following prosthesis causes: vascular compromise, aseptic loosening, periprosthetic fractures, and metallosis. Sarcoma was associated with a higher infection rate. Radiotherapy and chemotherapy (not in combination) were statistically associated with an increased infection. Debridement with retention of the implant has reached a remission of the infection rate of $70 \%, 62 \%$ for two-step treatment to $100 \%$ in the case of amputations.

Conclusions. Regardless of the stage of the tumor, amputation has a narrower indication nowadays, the goal being the retention of the limb and reconstruction.

The amputation post-tumoral arthroplasty is of a primary intention in the case of aggressive local recurrences and massive infections. Staphylococcus aureus remains the bacterium with the highest incidence of infection complications.

Keywords: tumoral arthroplasty, post-intervention amputation, local recurrence 